\title{
Controlled crack propagation for atomic precision handling of wafer-scale two-dimensional materials
}

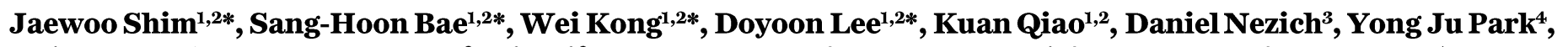
Ruike Zhao $^{1,5}$, Suresh Sundaram ${ }^{6}$, Xin Li $^{6}$, Hanwool Yeon ${ }^{1,2}$, Chanyeol Choi ${ }^{1,2}$, Hyun Kum ${ }^{1,2}$, Ruoyu Yue ${ }^{7}$, Guanyu Zhou ${ }^{7}$, Yunbo Ou ${ }^{8}$, Kyusang Lee ${ }^{1,2,9}$, Jagadeesh Moodera $^{8}$, Xuanhe Zhao ${ }^{1}$, Jong-Hyun Ahn ${ }^{4}$, Christopher Hinkle $^{7,10}$, Abdallah Ougazzaden ${ }^{6}$, Jeehwan Kim ${ }^{1,2,11,12 \uparrow}$

${ }^{1}$ Mechanical Engineering, Massachusetts Institute of Technology, Cambridge, MA, USA. ${ }^{2}$ Research Laboratory of Electronics, Massachusetts Institute of Technology, Cambridge, MA, USA. 'Lincoln Laboratory, Massachusetts Institute of Technology, Lexington, MA, USA. ${ }^{4}$ School of Electrical and Electronic Engineering, Yonsei University, Seoul 03722, Republic of Korea. ${ }^{5}$ Department of Mechanical and Aerospace Engineering, The Ohio State University, Columbus, OH 43210, USA. ${ }^{6}$ School of Electrical and Computer Engineering, Georgia Institute of Technology, UMI 2958 GT-CNRS, GT-Lorraine, Metz, France. ${ }^{7}$ Materials Science and Engineering, University of Texas at Dallas, Richardson, TX, USA. ${ }^{8}$ Department of Physics, and Plasma Science and Fusion Center, Massachusetts Institute of Technology, Cambridge, MA, USA. ${ }^{9}$ Departments of Electrical \& Computer Engineering and Materials Science Engineering, University of Virginia, Charlottesville, VA, USA. ${ }^{10}$ Department of Electrical Engineering, University of Notre Dame, Notre Dame, IN, USA. ${ }^{11}$ Materials Science and Engineering, Massachusetts Institute of Technology, Cambridge, MA, USA. ${ }^{12 M i c r o s y s t e m ~ T e c h n o l o g y ~}$ Laboratory, Massachusetts Institute of Technology, Cambridge, MA, USA.

*These authors contributed equally to this work.

†Corresponding author. Email: jeehwan@mit.edu

Although flakes of two-dimensional (2D) heterostructures at micrometer scale can be formed with adhesive-tape methods, isolation of $2 \mathrm{D}$ flakes into monolayers is extremely time-consuming as it is a trialand-error process. Controlling the number of 2D layers through direct growth also presents difficulty because of the high nucleation barrier on 2D materials. We demonstrate a layer-resolved 2D material splitting technique that permits the high-throughput production of multiple monolayers of wafer-scale ( 5 centimeter diameter) 2D materials by splitting single stacks of thick 2D materials grown on a single wafer. Wafer-scale uniformity of $h-B N, W_{2}, W S e_{2}, M S_{2}$, and $\mathrm{MoSe}_{2}$ monolayers was verified by photoluminescence $(\mathrm{PL})$ response and by substantial retention of electronic conductivity. We fabricated wafer-scale van der Waals heterostructures, including field-effect transistors, with single-atom thickness resolution.
\end{abstract}

Because of their in-plane stability and weak out-of-plane interaction, two-dimensional (2D) materials can be stacked together to form a multitude of device types with a broad spectrum of functionalities (1-6). Construction of 2D material-based heterostructures is often described as stacking Lego blocks (4). In order to tailor the 2D heterostructure characteristics for specific functionalities, it is essential to be able to isolate 2D materials into monolayer films and stack them with monolayer precision. The most common method for assembling these 2D blocks is by using the adhesive-tape method (7), where stacking of micrometer-scale flakes has been demonstrated (4). However, this method cannot reliably produce monolayer $2 \mathrm{D}$ crystals from bulk materials. The process becomes more complicated if the heterostructure design requires several different types of 2D material monolayers. Multiple monolayer flakes must be initially secured for each 2D material, which becomes extremely time consuming. Moreover, although the isolation of flakes into nominal monolayer has been demonstrated, the lateral dimensions (hundreds of micrometers) are not sufficient to guarantee the fabrication of large-scale 2D heterostructures (8). In parallel, numerous efforts have been made to directly grow 2D heterostructures at the wafer scale $(6,9,10)$. Recently, metal-organic chemical vapor deposition (MOCVD) growths of waferscale monolayer 2D materials and their heterostructures have been successfully demonstrated for some of transition metal dichalcogenides (TMDCs) at a specific growth condition (11, 12).

We introduce a layer-resolved splitting (LRS) technique that can be universally applied to produce $2 \mathrm{D}$ material monolayers at the wafer scale. This method requires one short growth of thick 2D materials on the wafer at a relaxed growth condition and subsequently harvests the multilayers to individual monolayers through a wafer-scale splitting process. This method allows for the high-throughput production of monolayer 2D materials with single-atom thickness precision for the fabrication of wafer-scale van der Waals (vdW) heterostructures. We demonstrated the wafer-scale LRS for various monolayers of $2 \mathrm{D}$ materials, including hexagonal boron nitride $\left(h\right.$-BN), tungsten disulfide $\left(\mathrm{WS}_{2}\right)$, tungsten diselenide $\left(\mathrm{WSe}_{2}\right)$, molybdenum disulfide $\left(\mathrm{MoS}_{2}\right)$, and molybdenum diselenide $\left(\mathrm{MoSe}_{2}\right)$. These films are readily stackable for 
forming wafer-scale 2D heterostructures. We have revealed the underlying mechanics that allows precise control of crack propagation, enabling LRS of the $2 \mathrm{D}$ material multilayers into multiple individual monolayers. The wafer-scale monolayer of TMDCs after LRS exhibits substantial photoluminescence (PL) enhancement uniformly across a 2-inch $(5-\mathrm{cm})$ wafer at a photon energy corresponding to its monolayer. Through this digital control of monolayer 2D materials, we demonstrate wafer-scale vdW heterostructures with singleatom thickness resolution. The heterostructure devices prepared by LRS and quasi-dry stacking process exhibited substantial improvement in electrical and optical uniformity across the wafer. This finding will open up new opportunities for $2 \mathrm{D}$ material research community as it provides a reliable pathway to form wafer-scale 2D heterostructures with monolayer resolution.

The schematic of the LRS process (Fig. 1A) shows a thick 2D material with an arbitrary number of layers grown on sapphire. Because of the difficulty in controlling the nucleation of the 2D material, new nucleation sites inevitably appear on top of the initial nucleation layer on the wafer before full substrate coverage. Thus, growths of multilayer 2D materials result in irregular, discontinuous films at the top, but does leave uniform continuous films underneath. Once the entire multilayer film is removed from the sapphire wafer, the continuous 2D material films on the bottom can be split into many monolayers with the LRS process. We designed the LRS process based on differences in the interfacial toughness $(\Gamma)$ of these materials. We used a 600-nm-thick nickel (Ni) film as an atomic-scale adhesive because the reported $\Gamma$ between $2 \mathrm{D}$ materials and $\mathrm{Ni}\left(\Gamma_{2 D-N i} \sim 1.4 \mathrm{~J} \mathrm{~m}^{-2}\right)(13)$ is three times greater than that of the vdW interface between layers in 2D materials $\left(\Gamma_{2 D-2 D}=0.45 \mathrm{~J} \mathrm{~m}^{-2}\right)(14)$. The $\Gamma$ between $2 \mathrm{D}$ materials and sapphire $\left(\Gamma_{2 D \text {-Sapphire }}\right)$ has been empirically deduced (see methods) and its average value $\left(\Gamma_{2 D \text {-Sapphire }}=0.26 \mathrm{~J} \mathrm{~m}^{-2}\right)$ is less than $\Gamma_{2 D-2 D}$. Application of a bending moment during the liftoff of a Ni/2D material stack on sapphire supplies elastic strain energy to the bottom interfaces (Fig. 1B). The strain energy per unit area is released upon delamination when reaching to desired strain release rate $(G)$. As shown in Fig. $1 C$, an external force creates a bending moment, resulting in a torque applied across a small distance in the sample that initiates spalling mode fracture where the cracks propagate downward due to mixed mode I and mode II fracture (15). 2D materials grown at the edge of the wafer is typically defective so that the crack propagation can be facilitated. Spalling mode fracture occurs due to the external bending moment applied. In addition to the opening mode stress (mode I) acting on the crack tip, a shear field (mode II) is created to guide the cracks into the bulk. Thus, exfoliation of $\mathrm{Ni} / 2 \mathrm{D}$ material stacks can separate the 2D-sapphire interface that has the weakest interfacial toughness and allow clean separation of
2D materials from the wafer.

We first applied the LRS technique to 4-nm-thick $\mathrm{WS}_{2}$ grown on a sapphire wafer through vapor phase epitaxy (VPE), characterizing each step of the process. As shown in the Raman mapping of as-grown $\mathrm{WS}_{2}$ on the sapphire wafer, the $E_{2 \mathrm{~g}}^{1}$ peak of the $\mathrm{WS}_{2}$ indicates that the sapphire wafer is fully covered with $\mathrm{WS}_{2}$ after the growth (see Fig. 1D and fig. S1). Atomic force microscopy (AFM) topology taken from the top of the as-grown $\mathrm{WS}_{2}$ (Fig. $1 \mathrm{E}$ ) shows that a rough discontinuous surface morphology developed from uncontrollable nucleation during growth, which is typically observed for the as-grown $2 \mathrm{D}$ materials. The LRS process was initiated with an exfoliation step to remove the entire $\mathrm{WS}_{2}$ stack from the sapphire wafer. A second exfoliation step isolated them into monolayer films. For the first exfoliation, a 600-nm-thick Ni film was deposited on multilayer $\mathrm{WS}_{2}$ followed by application of thermal release tape on the $\mathrm{Ni}$ as a handler. Lifting off the tape/Ni stack successfully separated the weakest $\mathrm{WS}_{2}$ sapphire interface resulting in release of the entire $\mathrm{WS}_{2}$ film from the substrate; no sign of $\mathrm{WS}_{2}$ was detected from Raman mapping and x-ray photoelectron spectroscopy on the sapphire wafer after exfoliation (see Fig. 1F and figs. S2 and S3). Thus, a moment applied by lift-off supplies strain energy to the $\mathrm{Ni} / \mathrm{WS}_{2}$ stacks sufficient to delaminate the weakest 2Dsapphire interface (16-18). Successful release of the entire $\mathrm{WS}_{2}$ film left pristine $\mathrm{WS}_{2}$ layers at the bottom. The bottom $\mathrm{WS}_{2}$ layer was continuous and smooth with root mean square (RMS) roughness of $0.5 \mathrm{~nm}$ (an average value measured from 15 locations, see Fig. 1G) that was the result of complete merging of the nuclei of the initial layers.

To harvest the continuous $\mathrm{WS}_{2}$ monolayer, we deposited a Ni layer on the bottom of the $\mathrm{WS}_{2}$ film while retaining the top tape/Ni/WS ${ }_{2}$ stack as-exfoliated (see Fig. 1A). Similar to peeling off the $\mathrm{Ni} / \mathrm{WS}_{2}$ stack from the sapphire substrate, we applied a moment from the top Ni to initiate spalling mode fracture for guiding the cracks downward. As $\Gamma_{2 D-N i}$ is substantially higher than $\Gamma_{2 D-2 D}$, the cracks arriving near the bottom $\mathrm{Ni}$ propagated through weaker $\mathrm{WS}_{2}-\mathrm{WS}_{2}$ interface directly above the bottom Ni layer (see Fig. 1C). Thus, the $\mathrm{Ni} / \mathrm{WS}_{2}$ stack separated upon peeling while the bottom $\mathrm{Ni}$ strongly adhered to the $\mathrm{WS}_{2}$ monolayer, which left a monolayer of $\mathrm{WS}_{2}$ on the bottom Ni layer.

We transferred this monolayer film onto an 8-inch (20.3cm) Si wafer coated with $90-\mathrm{nm}$ of $\mathrm{SiO}_{2}$ to investigate the thickness and quality of the exfoliated monolayer $\mathrm{WS}_{2}$ film (see fig. S4 for photographs of the entire LRS processes). As shown in Fig. 2, A and B, the 2-inch wafer-scale $\mathrm{WS}_{2}$ transferred intact onto the 8-inch $\mathrm{SiO}_{2} / \mathrm{Si}$ wafer without prominent wrinkle, folding, or ripple. The average thickness of the transferred $\mathrm{WS}_{2}$ is $0.7 \mathrm{~nm}$ (average value obtained from scans on 10 locations, Fig. $2 \mathrm{C}$ ). The exact value for the monolayer is $0.616 \mathrm{~nm}(19)$, and we speculate that the greater observed 
thickness arose tip-sample interactions under non-contact measurement mode. Successful isolation of $\mathrm{WS}_{2}$ monolayer was confirmed by the substantial enhancement of peak intensity of photoluminescence (PL) spectra (Fig. 2D) at its direct gap of $1.99 \mathrm{eV}$, versus the weak and wide PL characteristic of a thick $\mathrm{WS}_{2}$ layer at its indirect gap of 1.97 $\mathrm{eV}(20-25)$. Also, the PL intensity was not further degraded (fig. S5), implying that $\mathrm{WS}_{2}$ was already degraded before the process given the prolonged time period after growth, which is typically observed in TMDCs after air exposure (26). Moreover, the strong PL intensity originating from the monolayer isolation was uniform across the entire 2-inch wafer area (spatial resolution of $2 \mathrm{~mm}$, Fig. 2E). PL mapping with greater spatial resolution further confirmed uniform monolayer thickness obtained by LRS (see Fig. $2 \mathrm{~F}$ for $50 \mu \mathrm{m}$ resolution map on a $1 \mathrm{~mm} \times 1 \mathrm{~mm}$ area and fig. $\mathrm{S} 6$ for $1 \mu \mathrm{m}$ resolution maps on $20 \mu \mathrm{m} \times 20 \mu \mathrm{m}$ area). Wafer-scale monolayer thickness was also confirmed by mapping the PL peak position where peaks are all concentrated at its direct gap of $1.99 \mathrm{eV}$ (see fig. S7) (20-25).

All LRS $\mathrm{WS}_{2}$ films were transferred with a quasi-dry process, where the $\mathrm{WS}_{2}$ split from the multilayers was directly dry-bonded to the $\mathrm{SiO}_{2}$-coated $\mathrm{Si}$ wafer followed by etching to remove the $\mathrm{Ni}(16,17,27)$. The average RMS roughness was $0.5 \mathrm{~nm}$ after LRS measured from 15 different spots in each material (see fig. S8). We speculate that perfect atomic smoothness (RMS $<0.3 \mathrm{~nm}$ ) was not obtained because of the polycrystalline nature of the films with a grain size of $\sim 60 \mathrm{~nm}$ (see fig. S9). Larger-scale morphological inspection of monolayer 2D materials after LRS with Raman microscopy and laser scanning confocal microscopy revealed no prominent surface defects and macroscopic deformation such as wrinkle, folding, or ripple (see figs. S10 and S11). More importantly, LRS did not substantially degrade electrical quality of the $2 \mathrm{D}$ materials. The average Hall mobility measured from few layers before LRS was $106.8 \mathrm{~cm}^{2} \mathrm{~V}^{-1} \mathrm{~s}^{-1}$ versus was $89.5 \mathrm{~cm}^{2} \mathrm{~V}^{-1} \mathrm{~s}^{-1}$ for the monolayer after LRS (see fig. S12) (28).

The LRS process was repeated to harvest additional continuous monolayers until the split layer was no longer continuous. In parallel, an entire 4-nm-thick $\mathrm{WS}_{2}$ film was transferred from another sapphire substrate onto a 90-nmthick $\mathrm{SiO}_{2}$-coated $\mathrm{Si}$ as a reference. Optical micrograph and scanning electron microscope (SEM) image seen in Fig. 3, A and B, showed its non-uniform thickness. Repetition of LRS yielded continuous production of uniform continuous monolayer $\mathrm{WS}_{2}$ (see fig. S13 for Raman spectra showing right shift for $E_{2}^{1}$ g peak compared to that of as-exfoliated 4-nm-thick $\mathrm{WS}_{2}$ films) through three cycles, as shown in Fig. 3, C to $\mathrm{H}$. After the third cycle, residual $\mathrm{WS}_{2}$ was observed as discontinuous triangle domains on the top Ni film (see Fig. 3, I and J), indicating that the LRS process has reached the final top layer where nucleated islands failed to merge during growth.
Furthermore, we measured strong direct gap emission peaks (at $1.99 \mathrm{eV}$ ) only from the $\mathrm{WS}_{2}$ obtained through the first three cycles (see Fig. 3K).

We applied our LRS technique to other 2D materials including $\mathrm{MoSe}_{2}, \mathrm{WSe}_{2}, \mathrm{MoS}_{2}$, and $h$-BN, and confirmed successful monolayer splitting for all of these materials. As shown in fig. S14, 3-nm-thick $h$-BN grown on sapphire was split into three $h$-BN monolayers. In addition, as shown in fig. $\mathrm{S} 15$, monolayer isolation of $\mathrm{MoSe}_{2}, \mathrm{WSe}_{2}$, and $\mathrm{MoS}_{2}$ after LRS was confirmed by PL measurement (see also figs. S16 to S18 for optical microscope images and Raman spectra of $\mathrm{MoSe}_{2}$, $\mathrm{WSe}_{2}$, and $\mathrm{MoS}_{2}$ before and after LRS process). We also characterized these monolayers with AFM, confocal Raman microscopy and laser scanning confocal microscopy (figs. S8, S10, and S11).

We then fabricated various $2 \mathrm{D}$ heterostructures. First, we fabricated $\mathrm{WS}_{2} / h$-BN heterostructures with the monolayer $\mathrm{WS}_{2}$ sandwiched between $h$-BNs to investigate the effect of wafer-scale encapsulation of TMDCs. Reduction of carrier scattering by surface optical phonon has been observed in the flakes of $h$-BN/TMDC/ $h$-BN heterostructures (29). Waferscale $h$-BN/TMDC/h-BN heterostructures were fabricated with LRS together with the quasi-dry transfer process. Monolayer $h$-BN prepared by LRS was transferred twice on a $\mathrm{SiO}_{2} / \mathrm{Si}$ wafer followed by monolayer transfer of $\mathrm{WS}_{2}$ on top, and capped by transferring $h$-BN twice for encapsulation (see fig. S19 for the schematic description of 2D heterostructure via LRS process). We observed a substantial enhancement of PL intensity uniformly across the wafer $(1 \mathrm{~mm} \times 1 \mathrm{~mm}$ PL map shown in Fig. 4A and measured across the wafer with same PL results), compared to that of a $\mathrm{WS}_{2}$ monolayer quasidry transferred on $\mathrm{SiO}_{2}$ (see Fig. 4B).

When we fabricated $h$-BN/ $\mathrm{WS}_{2} / h$-BN heterostructures using a wet-stacking process by scooping $2 \mathrm{D}$ materials from a solution (see method for details of the wet-stacking process), we observed a substantially weakened PL response compared to that of heterostructures prepared by the quasi-dry-stacking process (see Fig. 4C), which were attributed to interference coming from poly(methyl methacrylate) (PMMA) residues at the interface (see fig. S20). Representative PL spectra for monolayer $\mathrm{WS}_{2}$ on $\mathrm{SiO}_{2}$, and double-layer $h$ $\mathrm{BN} /$ monolayer $\mathrm{WS}_{2}$ /double-layer $h$-BN prepared by quasidry- and wet-stacking are shown in Fig. 4E. Substantial degradation of the PL intensity was observed for the wet-stacked $h$-BN/ $\mathrm{WS}_{2}$ heterostructure even compared to quasi-dry-transferred $\mathrm{WS}_{2}$ on $\mathrm{SiO}_{2}$. A 15-fold enhancement of PL intensity was observed for $h$-BN/WS $/ h$-BN quasi-dry-stacks compared to that for $\mathrm{WS}_{2}$ wet-stacks on $\mathrm{SiO}_{2}$ (see Fig. 4D).

Our study revealed wafer-scale enhancement in optoelectronic quality of monolayer $\mathrm{WS}_{2}$ by wafer-scale $h$-BN encapsulation that should permit fabrication of large-scale optoelectronic devices based on van der Waals 
heterostructures. Our quasi-dry stacking method is the key to achieving optoelectronic enhancement in TMDCs by $h$-BN encapsulation as the process preserves the quality of the $2 \mathrm{D}$ materials. The optical quality of the TMDCs was not severely affected, although the roughness of the surface slightly increases with increasing layer numbers in the heterostructures (see fig. S21) possibly because of the presence of nanometer-scale Ni residues after quasi-dry stacking process (17).

We also fabricated arrays of 2D heterostructure devices at the wafer scale. These heterostructure arrays exhibited uniformly enhanced device performance across the wafer. LRS fabricated $\mathrm{MoS}_{2}$ and $h$-BN multilayers were quasi-dry-stacked on a $\mathrm{SiO}_{2} / \mathrm{Si}$ wafer to form field effect transistors (FETs). Deleterious trap charges from the substrate were avoided by using $h$-BN (see Fig. 4F for the structure of our 2D heterostructure device) (30-32). Figure 4G shows an optical image of the $10 \times 10$ arrays of $\mathrm{MoS}_{2}$ FETs on a $1-\mathrm{cm}^{2}$ wafer. Although both FETs with and without $h$-BN exhibited high on-off ratios of $>10^{7}$ (see fig. S22), the FETs without $h$-BN shows very large hysteresis in its drain current-gate voltage sweep which is detrimental to its transistor operation. However, substantial suppression of hysteresis has been observed in FETs with $h$-BN (see Fig. 4H). This reduction of hysteresis was obtained uniformly across the arrays in the wafer. As shown in the map of hysteresis of FETs with and without $h$ $\mathrm{BN}$, the FET arrays with $h$-BN showed excellent reduction in hysteresis during a gate voltage sweep applied uniformly across the wafer (see Fig. 4I).

The merit of quasi-dry stacking process was also highlighted by fabricating arrays of $\mathrm{WSe}_{2} /$ graphene vertical transistors (see fig. S23 for an optical image of the $8 \times 8$ arrays of $\mathrm{WSe}_{2} /$ graphene vertical transistors made on a $1 \mathrm{~cm} \times 1 \mathrm{~cm}$ wafer). As shown in the map of on/off ratios of vertical transistors (fig. S23), our transistor arrays made by quasi-dry stacking showed excellent device-to-device uniformity with 9.6\% variation (see fig. S24 for the statistical distribution). Vertical transistor arrays fabricated by the wet-stacking process had a device-to-device variation of $26 \%$.

We have demonstrated that the LRS technique can precisely isolate monolayers of $2 \mathrm{D}$ materials at the wafer scale from multilayers of $2 \mathrm{D}$ materials, thus allowing wafer-scale integration of $2 \mathrm{D}$ materials. LRS permits high throughput manufacturing of wafer-scale monolayers of various $2 \mathrm{D}$ materials from thick 2D material films grown at relaxed growth conditions. We have demonstrated various 2D heterostructure devices at the wafer by applying LRS together with quasi-dry stacking. These heterostructure devices exhibit uniform device performances across the wafer. This high throughput manufacturing of $2 \mathrm{D}$ heterostructures will become the stepping stone for the commercialization of 2D material-based devices.

\section{REFERENCES AND NOTES}

1. T. Georgiou, R. Jalil, B. D. Belle, L. Britnell, R. V. Gorbachev, S. V. Morozov, Y.-J. Kim, A. Gholinia, S. J. Haigh, O. Makarovsky, L. Eaves, L. A. Ponomarenko, A. K. Geim, K. S. Novoselov, A. Mishchenko, Vertical field-effect transistor based on graphene-WS 2 heterostructures for flexible and transparent electronics. Nat. Nanotechnol. 8, 100-103 (2013). doi:10.1038/nnano.2012.224 Medline

2. L. Britnell, R. M. Ribeiro, A. Eckmann, R. Jalil, B. D. Belle, A. Mishchenko, Y.-J. Kim, R. V. Gorbachev, T. Georgiou, S. V. Morozov, A. N. Grigorenko, A. K. Geim, C. Casiraghi, A. H. Castro Neto, K. S. Novoselov, Strong light-matter interactions in heterostructures of atomically thin films. Science 340, 1311-1314 (2013). doi:10.1126/science. 1235547 Medline

3. J. Shim, S. Oh, D.-H. Kang, S.-H. Jo, M. H. Ali, W.-Y. Choi, K. Heo, J. Jeon, S. Lee, M. Kim, Y. J. Song, J.-H. Park, Phosphorene/rhenium disulfide heterojunction-based negative differential resistance device for multi-valued logic. Nat. Commun. 7, 13413 (2016). doi:10.1038/ncomms 13413 Medline

4. A. K. Geim, I. V. Grigorieva, Van der Waals heterostructures. Nature 499, 419-425 (2013). doi:10.1038/nature12385 Medline

5. C. H. Lee, G.-H. Lee, A. M. van der Zande, W. Chen, Y. Li, M. Han, X. Cui, G. Arefe, C. Nuckolls, T. F. Heinz, J. Guo, J. Hone, P. Kim, Atomically thin p-n junctions with van der Waals heterointerfaces. Nat. Nanotechnol. 9, 676-681 (2014). doi:10.1038/nnano.2014.150 Medline

6. Y. Gong, J. Lin, X. Wang, G. Shi, S. Lei, Z. Lin, X. Zou, G. Ye, R. Vajtai, B. I. Yakobson, H. Terrones, M. Terrones, B. K. Tay, J. Lou, S. T. Pantelides, Z. Liu, W. Zhou, P. M. Ajayan, Vertical and in-plane heterostructures from WS2/MoS2 monolayers. Nat. Mater. 13, 1135-1142 (2014). doi:10.1038/nmat4091 Medline

7. K. S. Novoselov, A. K. Geim, S. V. Morozov, D. Jiang, Y. Zhang, S. V. Dubonos, I. V. Grigorieva, A. A. Firsov, Electric field effect in atomically thin carbon films. Science 306, 666-669 (2004). doi:10.1126/science.1102896 Medline

8. S. B. Desai, S. R. Madhvapathy, M. Amani, D. Kiriya, M. Hettick, M. Tosun, Y. Zhou, M. Dubey, J. W. Ager 3rd, D. Chrzan, A. Javey, Gold-Mediated Exfoliation of Ultralarge Optoelectronically-Perfect Monolayers. Adv. Mater. 28, 4053-4058 (2016). doi:10.1002/adma.201506171 Medline

9. S. M. Eichfeld, L. Hossain, Y.-C. Lin, A. F. Piasecki, B. Kupp, A. G. Birdwell, R. A. Burke, N. Lu, X. Peng, J. Li, A. Azcatl, S. McDonnell, R. M. Wallace, M. J. Kim, T. S. Mayer, J. M. Redwing, J. A. Robinson, Highly scalable, atomically thin WSez grown via metal-organic chemical vapor deposition. ACS Nano 9, 2080-2087 (2015). doi:10.1021/nn5073286 Medline

10. Y. C. Lin, R. K. Ghosh, R. Addou, N. Lu, S. M. Eichfeld, H. Zhu, M.-Y. Li, X. Peng, M. J. Kim, L.-J. Li, R. M. Wallace, S. Datta, J. A. Robinson, Atomically thin resonant tunnel diodes built from synthetic van der Waals heterostructures. Nat. Commun. 6. 7311 (2015). doi:10.1038/ncomms8311 Medline

11. K. Kang, S. Xie, L. Huang, Y. Han, P. Y. Huang, K. F. Mak, C.-J. Kim, D. Muller, J. Park, High-mobility three-atom-thick semiconducting films with wafer-scale homogeneity. Nature 520, 656-660 (2015). doi:10.1038/nature14417Medline

12. K. Kang, K.-H. Lee, Y. Han, H. Gao, S. Xie, D. A. Muller, J. Park, Layer-by-layer assembly of two-dimensional materials into wafer-scale heterostructures. Nature 550, 229-233 (2017). doi:10.1038/nature23905 Medline

13. Z. Xu, M. J. Buehler, Interface structure and mechanics between graphene and metal substrates: A first-principles study. J. Phys. Condens. Matter 22, 485301 (2010). doi:10.1088/0953-8984/22/48/485301 Medline

14. T. Björkman, A. Gulans, A. V. Krasheninnikov, R. M. Nieminen, van der Waals bonding in layered compounds from advanced density-functional first-principles calculations. Phys. Rev. Lett. 108, 235502 (2012). doi:10.1103/PhysRevLett.108.235502 Medline

15. Z. Suo, J. W. Hutchinson, Steady-state cracking in brittle substrates beneath adherent films. Int. J. Solids Struct. 25, 1337-1353 (1989). doi:10.1016/00207683(89) $90096-6$

16. J. Kim, H. Park, J. B. Hannon, S. W. Bedell, K. Fogel, D. K. Sadana, C. Dimitrakopoulos, Layer-resolved graphene transfer via engineered strain layers. Science 342, 833-836 (2013). doi:10.1126/science.1242988 Medline

17. S. H. Bae, X. Zhou, S. Kim, Y. S. Lee, S. S. Cruz, Y. Kim, J. B. Hannon, Y. Yang, D. K. Sadana, F. M. Ross, H. Park, J. Kim, Unveiling the carrier transport mechanism in epitaxial graphene for forming wafer-scale, single-domain graphene. Proc. Natl. Acad. Sci. U.S.A. 114, 4082-4086 (2017). doi:10.1073/pnas.1620176114 Medline 18. H. Rydberg, M. Dion, N. Jacobson, E. Schröder, P. Hyldgaard, S. I. Simak, D. C. 
Langreth, B. I. Lundqvist, Van der Waals density functional for layered structures. Phys. Rev. Lett. 91, 126402 (2003). doi:10.1103/PhysRevLett.91.126402 Medline

19. A. Molina-Sánchez, L. Wirtz, Phonons in single-layer and few-layer MoS2 and WS2. Phys. Rev. B 84, 155413 (2011). doi:10.1103/PhysRevB.84.155413

20. H. R. Gutiérrez, N. Perea-López, A. L. Elías, A. Berkdemir, B. Wang, R. Lv, F. LópezUrías, V. H. Crespi, H. Terrones, M. Terrones, Extraordinary room-temperature photoluminescence in triangular WS 2 monolayers. Nano Lett. 13, 3447-3454 (2013). doi:10.1021/nl3026357 Medline

21. J. Park, W. Lee, T. Choi, S.-H. Hwang, J. M. Myoung, J.-H. Jung, S.-H. Kim, H. Kim, Layer-modulated synthesis of uniform tungsten disulfide nanosheet using gasphase precursors. Nanoscale 7, 1308-1313 (2015). doi:10.1039/C4NR04292A Medline

22. S. Sasaki, Y. Kobayashi, Z. Liu, K. Suenaga, Y. Maniwa, Y. Miyauchi, Y. Miyata, Growth and optical properties of Nb-doped WS 2 monolayers. Appl. Phys. Express 9, 071201(2016). doi:10.7567/APEX.9.071201

23. N. J. Huo, Y. J. Yang, J. B. Li, Optoelectronics based on 2D TMDs and heterostructures. J. Semiconductors 38, 031002 (2017). doi:10.1088/16744926/38/3/031002

24. A. L. Elías, N. Perea-López, A. Castro-Beltrán, A. Berkdemir, R. Lv, S. Feng, A. D. Long, T. Hayashi, Y. A. Kim, M. Endo, H. R. Gutiérrez, N. R. Pradhan, L. Balicas, T. E. Mallouk, F. López-Urías, H. Terrones, M. Terrones, Controlled synthesis and transfer of large-area WS2 sheets: From single layer to few layers. ACS Nano 7 , 5235-5242 (2013). doi:10.1021/nn400971k Medline

25. F. Lan, R. Yang, Y. Xu, S. Qian, S. Zhang, H. Cheng, Y. Zhang, Synthesis of LargeScale Single-Crystalline Monolayer WS2 Using a Semi-Sealed Method. Nanomaterials 8, 100 (2018). doi:10.3390/nano8020100 Medline

26. Z. He, X. Wang, W. Xu, Y. Zhou, Y. Sheng, Y. Rong, J. M. Smith, J. H. Warner, Revealing Defect-State Photoluminescence in Monolayer WS 2 by Cryogenic Laser Processing. ACS Nano 10, 5847-5855 (2016). doi:10.1021/acsnano.6b00714 Medline

27. Y. Kim, S. S. Cruz, K. Lee, B. O. Alawode, C. Choi, Y. Song, J. M. Johnson, C. Heidelberger, W. Kong, S. Choi, K. Qiao, I. Almansouri, E. A. Fitzgerald, J. Kong, A. M. Kolpak, J. Hwang, J. Kim, Remote epitaxy through graphene enables twodimensional material-based layer transfer. Nature 544, 340-343 (2017). doi:10.1038/nature22053 Medline

28. H. Schmidt, F. Giustiniano, G. Eda, Electronic transport properties of transition metal dichalcogenide field-effect devices: Surface and interface effects. Chem. Soc. Rev. 44, 7715-7736 (2015). doi:10.1039/C5CS00275C Medline

29. S. M. Kim, A. Hsu, M. H. Park, S. H. Chae, S. J. Yun, J. S. Lee, D.-H. Cho, W. Fang, C. Lee, T. Palacios, M. Dresselhaus, K. K. Kim, Y. H. Lee, J. Kong, Synthesis of largearea multilayer hexagonal boron nitride for high material performance. Nat. Commun. 6, 8662 (2015). doi:10.1038/ncomms9662 Medline

30. G. H. Lee, X. Cui, Y. D. Kim, G. Arefe, X. Zhang, C.-H. Lee, F. Ye, K. Watanabe, T. Taniguchi, P. Kim, J. Hone, Highly Stable, Dual-Gated MoS2 Transistors Encapsulated by Hexagonal Boron Nitride with Gate-Controllable Contact, Resistance, and Threshold Voltage. ACS Nano 9, 7019-7026 (2015). doi:10.1021/acsnano. 5 b01341 Medline

31. M. Choi, Y. J. Park, B. K. Sharma, S.-R. Bae, S. Y. Kim, J.-H. Ahn, Flexible activematrix organic light-emitting diode display enabled by $\mathrm{MoS}_{2}$ thin-film transistor. Sci. Adv. 4, s8721 (2018). doi:10.1126/sciadv aas8721 Medline

32. M. Y. Chan, K. Komatsu, S.-L. Li, Y. Xu, P. Darmawan, H. Kuramochi, S. Nakaharai, A. Aparecido-Ferreira, K. Watanabe, T. Taniguchi, K. Tsukagoshi, Suppression of thermally activated carrier transport in atomically thin $\mathrm{MoS}_{2}$ on crystalline hexagonal boron nitride substrates. Nanoscale 5, 9572-9576 (2013). doi:10.1039/c3nr03220e Medline

33. X. Li, S. Sundaram, Y. El Gmili, T. Ayari, R. Puybaret, G. Patriarche, P. L. Voss, J. P. Salvestrini, A. Ougazzaden, Large-Area Two-Dimensional Layered Hexagonal Boron Nitride Grown on Sapphire by Metalorganic Vapor Phase Epitaxy. Cryst. Growth Des. 16, 3409-3415 (2016). doi:10.1021/acs.cgd.6b00398

34. R. Y. Yue, Y. Nie, L. A. Walsh, R. Addou, C. Liang, N. Lu, A. T. Barton, H. Zhu, Z. Che, D. Barrera, L. Cheng, P.-R. Cha, Y. J. Chabal, J. W. P. Hsu, J. Kim, M. J. Kim, L. Colombo, R. M. Wallace, K. Cho, C. L. Hinkle, Nucleation and growth of WSe2: Enabling large grain transition metal dichalcogenides. 2D Mater. 4, 045019 (2017). doi:10.1088/2053-1583/aa8ab5

\section{ACKNOWLEDGMENTS}

Funding: The author J. Kim acknowledge the support from NSF grant No. CMMI1825731. The author K. Lee acknowledge the support from NSF grant No. CMMI1825256. The authors A.O., S.S. and X.L. acknowledge the French National Research Agency for the co-funding of the $h$-BN study under the "GANEX" Laboratory of Excellence "LABEX" project. Author D.N. acknowledges the MIT Lincoln Laboratory Technology Office and Assistant Secretary of Defense for Research and Engineering for funding the growth of wafer-scale WS 2 films. J.M. acknowledge the support from NSF Grants DMR - 1700137, ONR Grant No. N00014-16-1-2657, and the STC Center for Integrated Quantum Materials under NSF Grant No. DMR-1231319. DISTRIBUTION STATEMENT A. Approved for public release. Distribution is unlimited. This material is based upon work supported by the Assistant Secretary of Defense for Research and Engineering under Air Force Contract No. FA8721-05-C-0002 and/or FA8702-15-D-0001. Any opinions, findings, conclusions or recommendations expressed in this material are those of the author(s) and do not necessarily reflect the views of the Assistant Secretary of Defense for Research and Engineering. Authors G.Z. and C.H. acknowledge that this work was supported in part by NEWLIMITS, a center in nCORE, a Semiconductor Research Corporation (SRC) program sponsored by NIST through award number 70NANB17H041. J. K. thanks to Prof. Jin-Hong Park of SKKU for fruitful discussion and support. J.K. acknowledge MIT-MI one-on-one project for partial support on this work. Author contributions: J.S., S.-H.B., W.K., and D.L. contributed equally to this work. J.K. conceived the idea, designed experiments, and directed the team. J.S., S.-H.B., W.K., D.L., K.Q., H.Y., C.C., H.K., and K.L. performed fabrication/characterization of all samples and wrote the manuscript. R.Z., Y.O., J.M., and X.Z. contributed to the computational model. D.N. worked on the growth of WS 2 on sapphire. S.S., X.L., and A.O. worked on the growth of $h$-BN on sapphire. R.Y., G.Z., and C.H. worked on the growth of $\mathrm{WSe}_{2}$ on sapphire. J.-H.A., and Y.J.P. worked on the growth of $\mathrm{MoS}_{2}$ on sapphire and transistor devices. All authors contributed to the discussion and analysis of the results regarding the manuscript. Competing interests: The authors declare no competing financial interests. Data and materials availability: All data needed to evaluate the conclusions in the paper are present in the paper or the supplementary materials.

\section{SUPPLEMENTARY MATERIALS}

www. sciencemag.org/cgi/content/full/science.aat8126/DC1 Materials and Methods

Figs. S1 to S24

References $(33,34)$

7 April 2018; resubmitted 11 August 2018

Accepted 21 September 2018

Published online 11 October 2018

10.1126/science.aat8126 
A

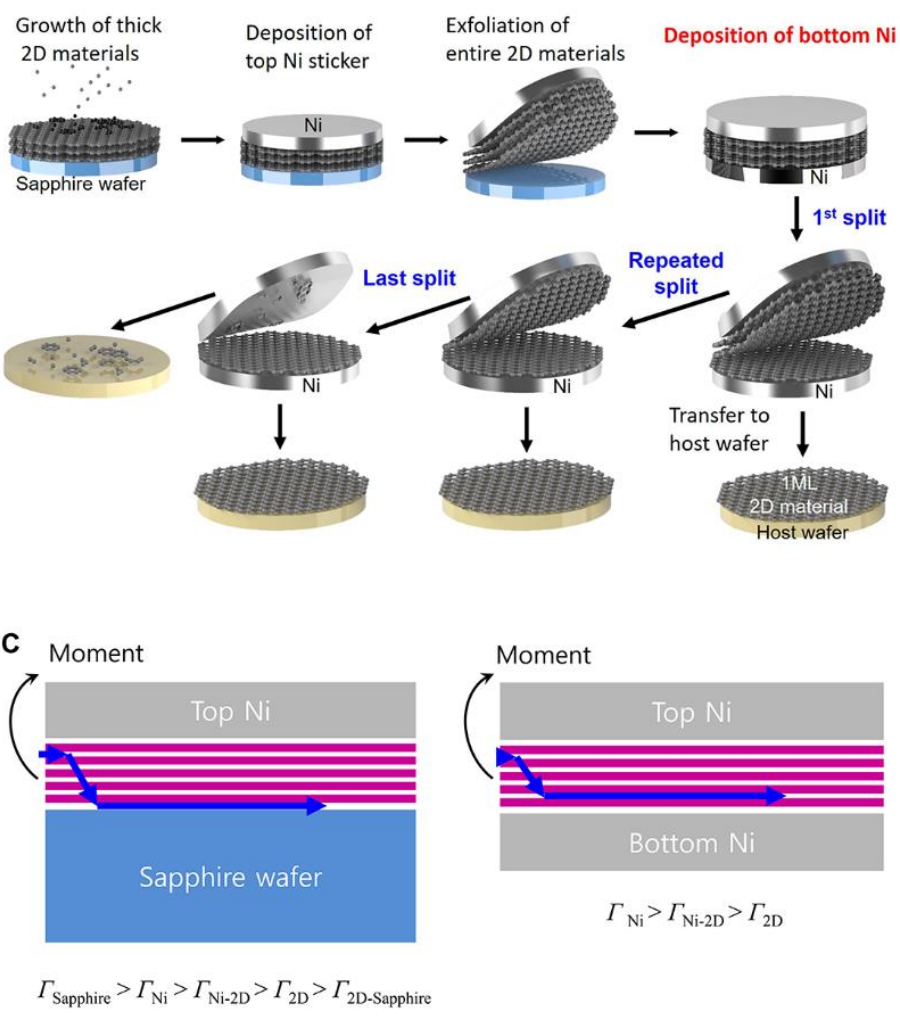

B
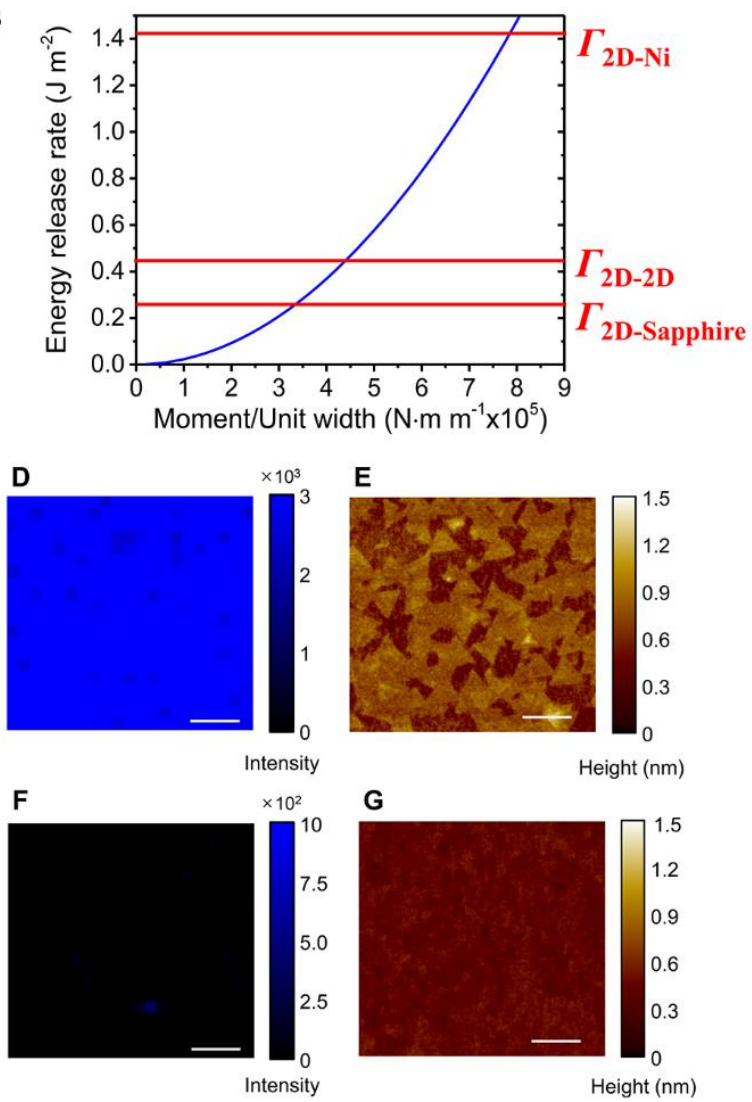

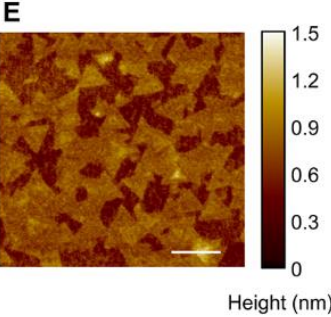

G

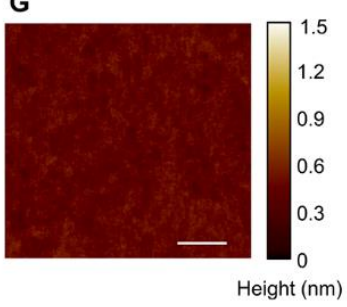

Fig. 1. Layer-resolved splitting (LRS) of 2D materials. (A) Schematic illustration explaining the LRS process of 2D materials. (B) modeling of energy release rate according to applied moment. (Ni thickness: $600 \mathrm{~nm}$ ) (C) Schematics of crack progression during LRS for first exfoliation of entire 2D materials from sapphire wafer (left) and exfoliation of the bottom monolayer 2D material (right). (D) Raman intensity mapping at $E_{2 \mathrm{~g}}$ peak $\left(353 \mathrm{~cm}^{-1}\right)$ of $W_{2}$ grown on a sapphire substrate, with laser wavelength and power of $532 \mathrm{~nm}$ and $2 \mathrm{~mW}$ in continuous waveform respectively, where the spatial resolution is $2 \mu \mathrm{m}$. (E) AFM topology taken from the top of as-grown 4$\mathrm{nm}$-thick WS $\mathrm{S}_{2}$ on the sapphire wafer. (F) Raman mapping image showing the intensity of $E_{2 \mathrm{~g}}$ peak $\left(353 \mathrm{~cm}^{-1}\right)$ on sapphire substrate after exfoliation of $\mathrm{WS}_{2}$ layer, with laser wavelength and power of $532 \mathrm{~nm}$ and $2 \mathrm{~mW}$, respectively. (G) AFM topology taken from the bottom of $W_{2}$ layer after exfoliation. Scale bars for Raman mapping images and AFM topology images are $2 \mu \mathrm{m}$ and $50 \mathrm{~nm}$, respectively. 
A

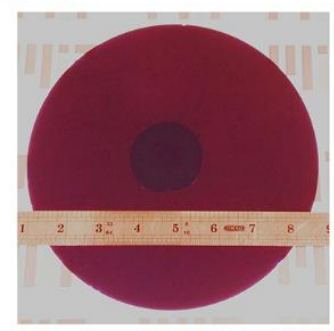

D

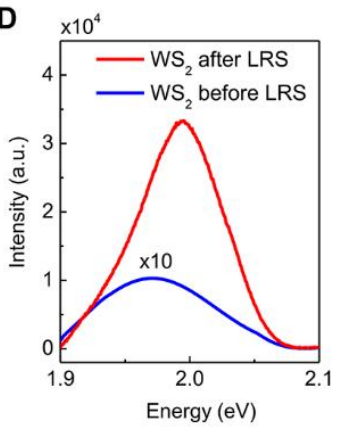

B

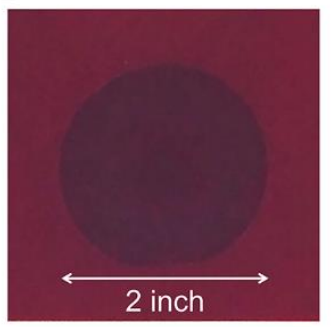

E

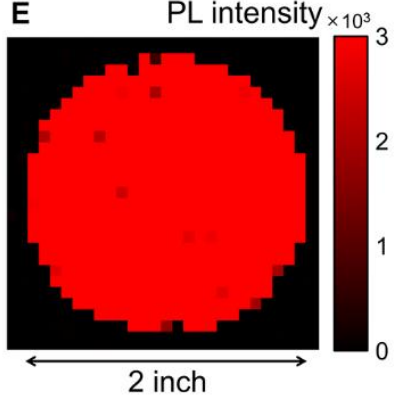

C

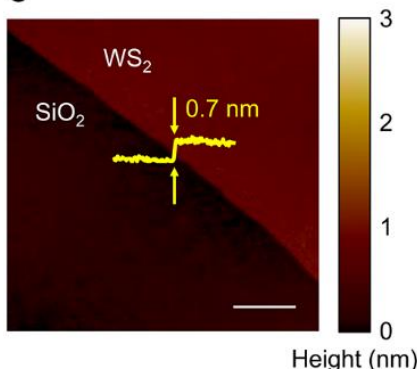

$\mathbf{F}$

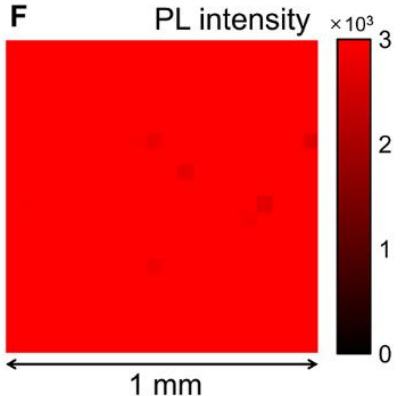

Fig. 2. Wafer-scale monolayer 2D material obtained by LRS process. (A) Optical image of 2-inch wafer-scale WS 2 monolayer on 8-inch oxidized $\mathrm{Si}$ wafer obtained through the LRS process. (B) Macrograph of 2-inch waferscale $\mathrm{WS}_{2}$ monolayer. (C) AFM image and height profile of the $\mathrm{WS}_{2}$ monolayer transferred on $\mathrm{SiO}_{2} / \mathrm{Si}$ wafer. Scale bar: $1 \mu \mathrm{m}$. (D) Representative PL spectra of as-exfoliated 4-nm-thick WS 2 (blue solid line) and monolayer WS $\mathrm{W}_{2}$ obtained by LRS process (red solid line), where the PL spectrum for monolayer $\mathrm{WS}_{2}$ is multiplied by 10 times to see the clear peak position. (E) Wafer-scale PL mapping image at $1.99 \mathrm{eV}$ of $\mathrm{WS}_{2}$ on the $\mathrm{SiO}_{2} / \mathrm{Si}$ wafer, where the spatial resolution is $2 \mathrm{~mm}$. (F) Large-scale $(1 \mathrm{~mm} \times 1 \mathrm{~mm})$ $\mathrm{PL}$ intensity map from $1.99 \mathrm{eV}$ for $\mathrm{WS}_{2}$ on $\mathrm{SiO}_{2}$, where the spatial resolution is $50 \mu \mathrm{m}$ (see fig. $\mathrm{S} 6$ for $1 \mu \mathrm{m}$ resolution map). All PL spectra were taken at the same laser power (2 $\mathrm{mW}$ ) and wavelength (532 nm). 

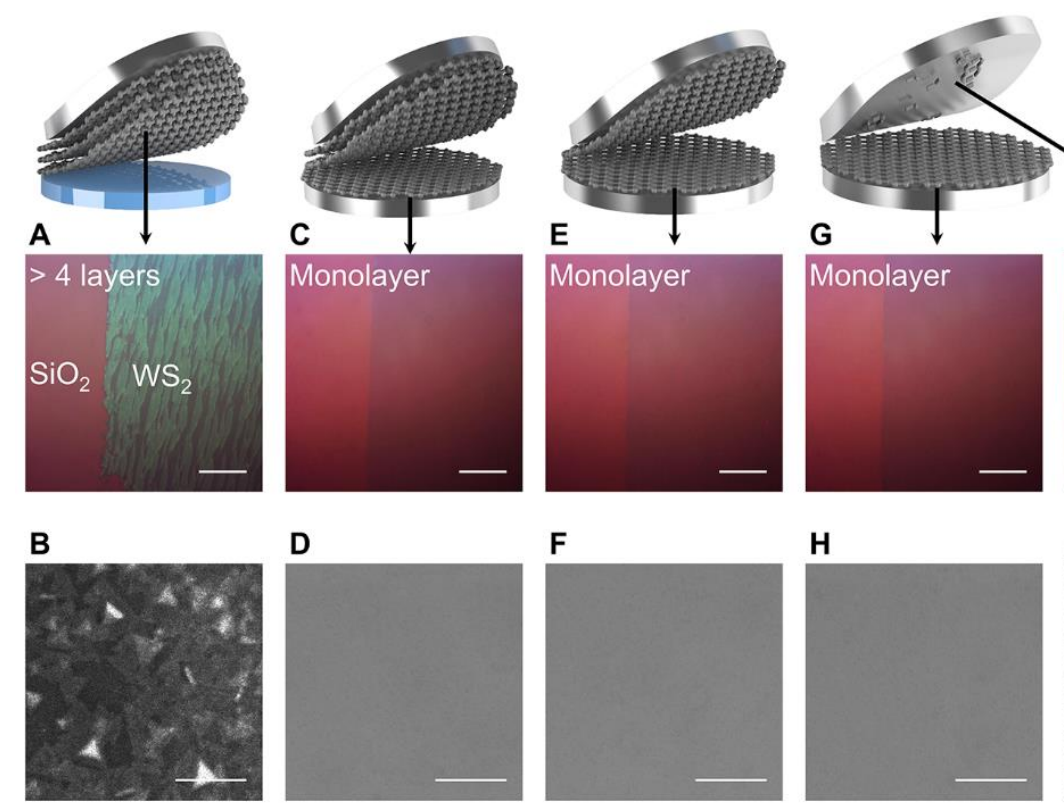

H

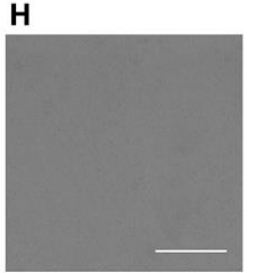

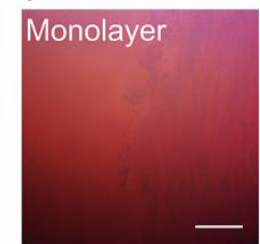

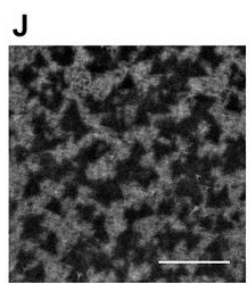

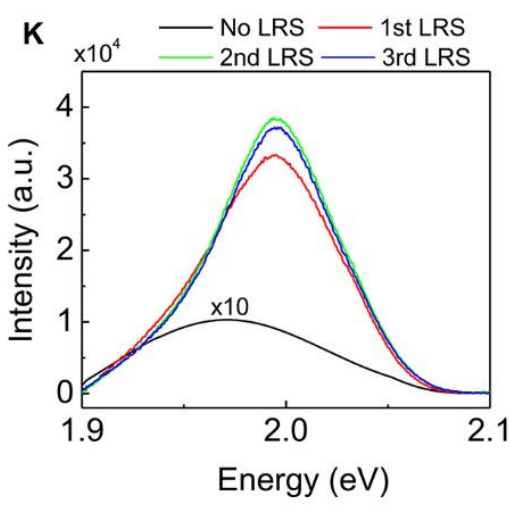

Fig. 3. Split of thick 2D materials into many monolayers via LRS process and their characterization. (A, C, E, G, and I) Optical micrographs and (B, D, F, H, and J) plan-view SEM images for as-exfoliated thick WS $(A)$ and $(B)$, monolayers of $W_{2}$ obtained by first $(C)$ and $(D)$, second $(E)$ and $(F)$, third $(G)$ and $(H)$, and the last $(I)$ and $(J)$ LRS processes, respectively. They are all transferred on 90-nm-thick $\mathrm{SiO}_{2} / \mathrm{Si}$ substrates. (K) Representative $\mathrm{PL}$ spectra for as-exfoliated thick WS 2 (black solid line) multiplied by ten times to see the clear peak position, monolayers of WS 2 obtained by first (red solid line), second (green solid line), third (blue solid line) LRS processes. All PL spectra were taken at the same laser power $(2 \mathrm{~mW})$ and wavelength (532 nm). Scale bars for optical microscopy images and plan-view SEM images are $50 \mu \mathrm{m}$ and $400 \mathrm{~nm}$, respectively. 

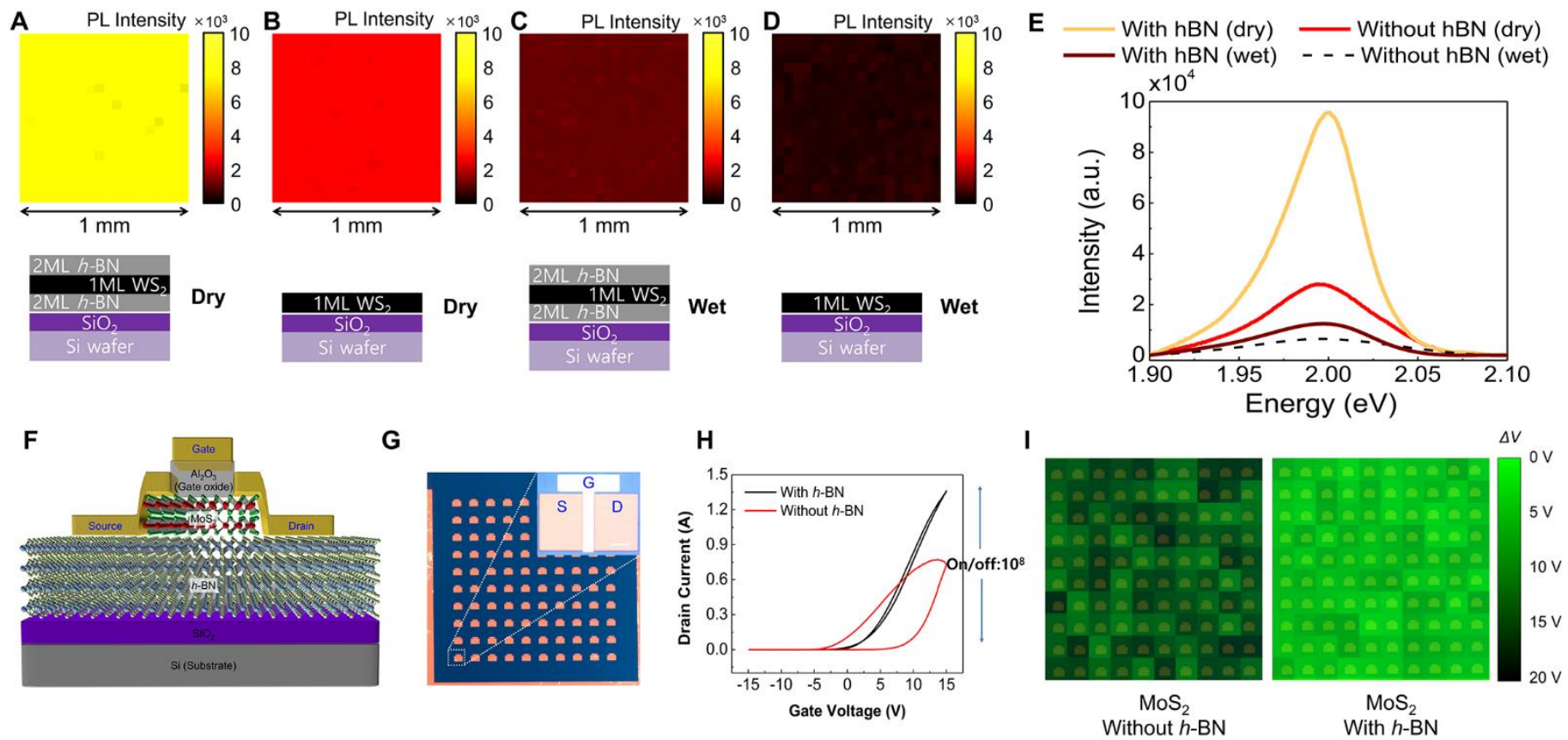

Fig. 4. Wafer-scale 2D heterostructures. (A) $\mathrm{PL}$ mapping images at $1.99 \mathrm{eV}$ of double-layer (2 $\mathrm{ML}) \mathrm{h}$ -

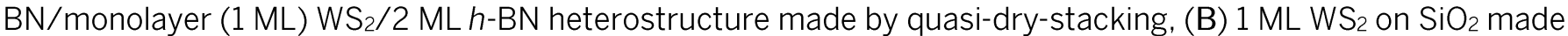
by quasi-dry-transfer, (C) $2 \mathrm{ML} h$-BN/1 ML WS $2 / 2 \mathrm{ML} h$-BN heterostructure made by wet-stacking, and (D) $1 \mathrm{ML}$ $W_{2}$ on $\mathrm{SiO}_{2}$ made by wet-stacking. (E) Representative PL spectra of all different structures made by different methods. All PL spectra were taken at the same laser power (2 mW) and wavelength (532 nm). (F) Schematic of a MoS2-based field effect transistor (FFT). (G) Macrograph of $10 \times 10 \mathrm{FET}$ arrays integrated on a $\mathrm{SiO}_{2} / \mathrm{Si}$ wafer with $1 \mathrm{~cm} \times 1 \mathrm{~cm}$ size. Inset shows micrograph of an individual device. Scale bar: $100 \mu \mathrm{m}$. The device area is defined by the gap between source and drain electrodes. $(\mathrm{H})$ Representative drain current-gate voltage $\left(I_{D}-V_{G}\right)$ characteristics of $\mathrm{MoS}_{2}$-based FETs at drain voltage $\left(V_{D S}\right)=1 \mathrm{~V}$. (I) $2 \mathrm{D}$ color maps of hysteresis voltage extracted from $I_{D}-V_{G}$ curves at $V_{D S}=1 V$ in transistor arrays made without $h$-BN (left) and with $h$-BN (right). 